\title{
Temperature-variable NMR Study of the keto-enol Tautomerism of Phenylpyruvic Acid
}

\author{
Toshiki YAMAJI ${ }^{\dagger}$ and Takeshi SAITo \\ National Metrology Institute of Japan (NMIJ), National Institute of Advanced Industrial Science and Technology \\ (AIST), Tsukuba, Ibaraki 305-8565, Japan
}

\begin{abstract}
The keto-enol tautomerism of phenylpyruvic acid (PPA) has been studied by temperature-variable $9.4 \mathrm{~T}$ nuclear magnetic resonance (NMR) spectroscopy. It was found that PPA favored its existence in the enol form in dimethyl sulfoxide (DMSO) solvent, $93.5 \%$ of the enol and 6.5\% of the keto tautomer was present in DMSO at room temperature. Increasing the temperature increased the percentage of the keto form. The experimental thermodynamic parameters for the tautomerism were successfully obtained from the spectral data.
\end{abstract}

Keywords Phenylpyruvic acid (PPA), keto-enol tautomerism, NMR, equilibrium constant, thermodynamic parameter

(Received July 4, 2017; Accepted September 25, 2017; Published February 10, 2018)

\section{Introduction}

Typical $\alpha$-keto acids, such as pyruvic acid (PA) and phenylpyruvic acid (PPA), are recognized to be biologically important metabolic products. ${ }^{1,2}$ PPA, which is the molecule studied in the present work, is an intermediate and catabolic byproduct of the phenylalanine metabolism in individuals afflicted with phenylketonuria (PKU). PKU is due to a lack of the enzyme phenylalanine hydroxylase (PAH), so that phenylalanine is converted not to tyrosine, but to PPA. PPA accumulates in the blood and tissues and is excreted in urine. The level of PPA is normally very low in blood or urine.

Many spectroscopic studies, ultra violet (UV), infrared (IR), Raman and nuclear magnetic resonance (NMR) studies, have been reported on the keto-enol tautomerism ${ }^{3-12}$ and conformation $^{6,9,10,12,13}$ of PA and PPA. These many investigations have revealed that these compounds exist as equilibrium mixtures consisting of the keto and enol forms. Here, the ketoenol tautomerism of PPA is shown in Scheme 1. Firstly, Larsen et al. found by ${ }^{1} \mathrm{H}$ NMR and UV spectroscopy that the enol isomer of PPA predominantly exists in dimethyl sulfoxide (DMSO), while PPA favors its existence in the keto form in $1 \mathrm{M}$ $\mathrm{HCl}^{3} \quad$ Sciacovelli et al. also investigated the tautomerism of PPA by NMR, UV, and IR spectroscopy, and found that the keto isomer increases in a water-DMSO solvent, depending upon the water content. ${ }^{4-6}$ Furthermore, PPA was found to predominantly take the keto form in the solid state and in an aqueous solution. Ray et al. analyzed the complete IR and Raman spectra of liquid and crystalline PA, and found that PA takes the cyclic

$\doteqdot$ To whom correspondence should be addressed.

E-mail: toshiki-yamaji@aist.go.jp

T. Y. present address: Spintronics Research Center, Theory Team, National Institute of Advanced Industrial Science and Technology (AIST), Central 2, 1-1-1 Umezono, Tsukuba, Ibaraki 305-8568, Japan. hydrogen-bonded dimer structure in which the two carbonyl groups are in a trans configuration in the pure liquid and crystalline phase. ${ }^{7}$ Next, Hanai et al. investigated the IR, Raman, and NMR spectra of PPA in the solid state, organic solvents and those its solid salts..$^{8-10}$ It was revealed that PPA takes the enol form in the solid state, and that the keto form is more stable in an aqueous solution. Carpy et al. assigned the structures of the two tautomeric forms of PPA in DMSO solvent by ${ }^{1} \mathrm{H}$ and ${ }^{13} \mathrm{C} \mathrm{NMR}$ spectroscopy, and found by $\mathrm{X}$-ray crystallography of the single crystal that the crystalline form takes the keto isomer. ${ }^{11,12}$ Moreover, the substituent effect on the tautomeric equilibrium was studied by using ab initio calculations in the gas phase, and the thermochemical parameters were obtained. They confirmed a large preference for the enol isomer relative to the keto isomer for PPA and $o$-chloro PPA. It was also found that the strong electron-withdrawing nitro group in o-nitro PPA has a significant influence on the keto-enol tautomerism, resulting in a shifting of the equilibrium towards the keto formation. Recently, Hanai et al. reported a polymorphism study of PPA on the basis of IR, Raman and solid state ${ }^{13} \mathrm{C}$ NMR spectroscopy, and revealed that two polymorph forms of PPA exist. ${ }^{13}$

NMR is perhaps the most important, powerful, and widely used form of spectroscopy in both academic and industrial chemical research. It provides the most detailed and unambiguous information on the chemical structure, functionality, and dynamics. We carried out $9.4 \mathrm{~T}$ solution NMR measurements as varying the temperature for the studying

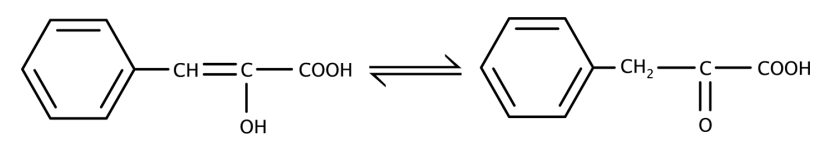

enol isomer

keto isomer

Scheme 1 keto-enol tautomerism of PPA. 


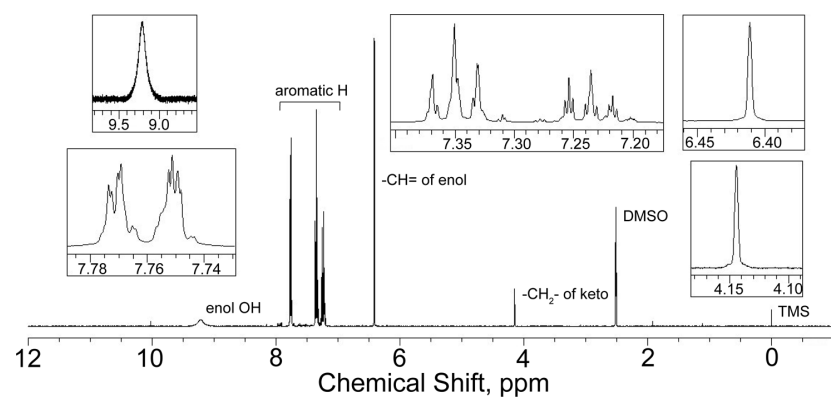

Fig. 1 9.4 T ${ }^{1} \mathrm{H}$ NMR experimental spectrum of PPA at room temperature. The solvent is DMSO. The inserted figure is the expanded region between 7 and $8 \mathrm{ppm}$, showing the signals of the aromatic protons.

the keto-enol tautomerism of PPA. The ${ }^{1} \mathrm{H}$ NMR spectra clearly showed that PPA favored its existence in the enol form in DMSO solvent. The equilibrium constant, conversion enthalpy and entropy for the tautomerism were obtained by this experimental study.

\section{Experimental}

A powder sample available from Tokyo Chemical Industry Co., Ltd was used to conduct $9.4 \mathrm{~T}{ }^{1} \mathrm{H}$ NMR measurements using a JEOL AL-400 NMR spectrometer. The acquisition time and the relaxation delay were 12.6 and $16.4 \mathrm{~s}$ for accurately estimating the intensity of the NMR signals, respectively. The scan times was 32. The chemical shift was measured with respect to the resonance frequency in tetramethylsilane (TMS). The solvent was DMSO. The concentration of PPA in DMSO was about $0.1 \mathrm{mM}$. For a structural analysis, except for the ${ }^{1} \mathrm{H}$ NMR spectrum reported in this paper, ${ }^{13} \mathrm{C}$ NMR, DEPT 135 (distorsionless enhancement by polarization transfer), HMQC (hetero-nuclear multiple quantum correlation spectroscopy), and HMBC (hetero-nuclear multiple bond correlation spectroscopy) measurements had also been conducted by the ame NMR spectrometer. Those data are not shown since they are outside the main topic of this study.

\section{Experimental Results}

The experimental ${ }^{1} \mathrm{H}$ NMR spectrum at room temperature is shown in Fig. 1. The expanded region between 7 and $8 \mathrm{ppm}$ is inserted in Fig. 1. An investigation of the ${ }^{1} \mathrm{H}$ NMR spectrum revealed the presence of a mixture of the enol and keto tautomers of PPA. The dissolution of PPA in a polar solvent, DMSO, can cause the tautomerization of the keto to the enol form. As can be seen in Fig. 1, the singlet peak at 4.14 ppm, the singlet peak at $6.41 \mathrm{ppm}$ and the broad peak at $9.2 \mathrm{ppm}$ can be assigned to the $-\mathrm{CH}_{2}$ - proton of the keto form, the $-\mathrm{CH}=$ proton of the enol form and the proton of the enol $\mathrm{OH}$, respectively. It can be concluded from the expanded region shown in Fig. 1 that the triplet peaks at $7.23 \mathrm{ppm}$, the triplet peaks at $7.35 \mathrm{ppm}$ and the doublet peaks at $7.76 \mathrm{ppm}$ account for the para-, meta- and ortho-protons, respectively. These signals between 7 and $8 \mathrm{ppm}$ consist of the peaks of the aromatic protons of both the enol and keto forms. It is known that the enol isomer with the $\mathrm{Z}$ configuration is dominantly present in the solution. ${ }^{4}$ The presence of the single peak due to the olefinic proton, even in

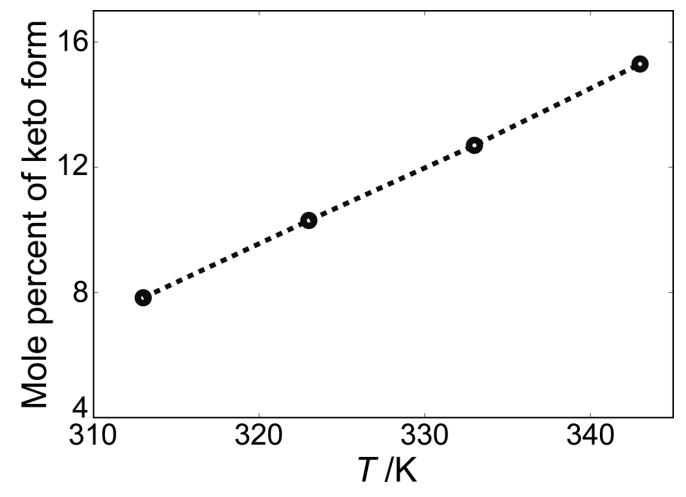

Fig. 2 Temperature dependence of the mole ratio of the keto form in the keto-enol tautomerism of PPA obtained from temperature-variable NMR measurements at above room temperature.

the expanded spectrum, points out the dominant formation of the enol form with the $\mathrm{Z}$ configuration.

The composition ratio of the keto and enol forms was precisely calculated from the relative peak-area integration of the $-\mathrm{CH}_{2}-$ proton of the keto form and the $-\mathrm{CH}=$ proton of the enol form, which were stable and observed at around the central region in the NMR spectrum. A peak-intensity calculation showed that this tautomeric mixture consisted of $93.5 \%$ of the enol form and $6.5 \%$ of the keto form. It can be considered that this enol form was remarkably stabilized by the intermolecular hydrogen bond between the hydrogen of the enol hydroxyl group and the DMSO molecule. ${ }^{5}$ This can also be seen from the fact that the $\mathrm{OH}$ proton of the enol form was more deshielded than an ordinary alcohol proton. It can be furthermore considered that PPA in DMSO, which is an aprotic solvent, is more stable in its enol form due to conjugation of the phenyl ring, the $\mathrm{C}=\mathrm{C}$ enol group and the carboxylic group. On the other hand, a protophilic solvent, such as water and $\mathrm{HCl}$, would reduce the enol formation due to the ionization of PPA. ${ }^{3-6,10}$ Upon considering the influence of a solvent on the keto-enol tautomerism on the basis of the Onsager model, ${ }^{14}$ the tautomer should favor its existence in the more polar isomer, i.e., the keto one as the polarity of a solvent increases. ${ }^{15}$ It can thereore be understood that the keto form is more stable than the enol one in another solvent, such as water and $\mathrm{HCl},{ }^{3-6,10}$ while the enol form becomes predominant in the DMSO solvent.

\section{Discussion}

It was found from ${ }^{1} \mathrm{H}$ NMR measurements that PPA exists as a tautomeric mixture of the enol and keto forms in a DMSO solution. Figure 2 shows the mole ratio of the keto form in the keto-enol tautomerism of PPA, obtained from temperaturevariable NMR measurements controlled by a thermostat system above room temperature. As can be seen in Fig. 2, increasing the temperature of this mixture increases the percentage of the keto form. It can be considered that this is the result from cleavage of the intermolecular hydrogen bond between the hydrogen of the enol hydroxyl group and the DMSO molecule owing to more activated molecular motion in the solution at high temperature. The equilibrium constant of the keto-enol tautomerism, $K_{\mathrm{T}}$, is defined as

$$
K_{\mathrm{T}}=\frac{[\text { enol form }]}{[\text { keto form }]}=\frac{\text { Peak intensity of } \mathrm{CH}}{0.5 \times\left(\text { peak intensity of } \mathrm{CH}_{2}\right)} .
$$


Table 1 Summary of tautomer equilibrium constant $K_{\mathrm{T}}$, and the mole ratio of the enol and keto form in the tautomerism of PPA

\begin{tabular}{crcc}
\hline$T / \mathrm{K}$ & $K_{\mathrm{T}}$ & Mole percent of enol Mole percent of keto \\
\hline Room temperature & 14.3 & 93.5 & 6.5 \\
313 & 11.8 & 92.2 & 7.8 \\
323 & 8.7 & 89.7 & 10.3 \\
333 & 6.9 & 87.3 & 12.7 \\
343 & 5.6 & 84.7 & 15.3 \\
\hline
\end{tabular}

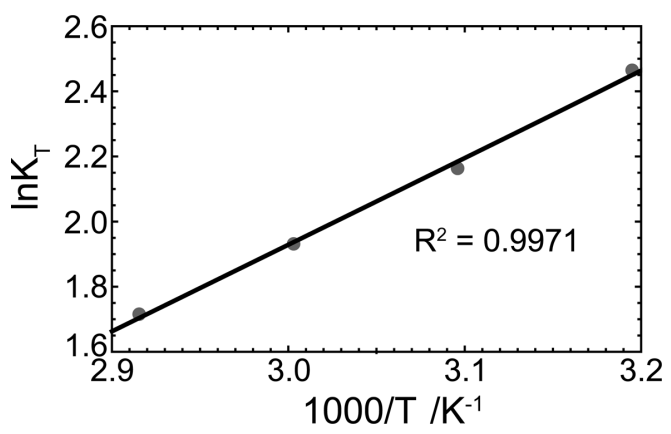

Fig. $3 \ln K_{\mathrm{T}}$ as a function of $(1 / T) \times 1000 . \Delta H^{0}$ and $\Delta S^{0}$ were obtained from the slope and the $y$-intercept of the line fitted on the plots, respectively.

The temperature dependence of the $K_{\mathrm{T}}$ experimentally obtained is summarized with the mole ratio of the keto and the enol form in the tautomerism of PPA in Table 1. The enthalpy, $\Delta H^{0}$, and entropy, $\Delta S^{0}$, for the conversion of the keto to enol form were obtained by the van't Hoff equation, as follows: ${ }^{11,16}$

$$
\ln K_{\mathrm{T}}=-\frac{\Delta H^{0}}{R}\left(\frac{1}{T}\right)+\frac{\Delta S^{0}}{R},
$$

where $R$ and $T$ are the molar gas constant and the temperature, respectively. Here, it was assumed that the values of the enthalpy and entropy for the tautomerism are independent from the temperature in the experimental range of this study. Figure 3 shows $\ln K_{\mathrm{T}}$ as a function of $1 / T . \Delta H^{0}$ and $\Delta S^{0}$ obtained from the regression line based on the least-squares method in Fig. 3 are $-22.2 \pm 0.85 \mathrm{~kJ} / \mathrm{mol}(-5.3 \pm 0.2 \mathrm{kcal} / \mathrm{mol})$ and $-50.5 \pm 2.6$ $\mathrm{kJ} /(\mathrm{mol} \mathrm{K}) \quad(-12.1 \pm 0.62 \mathrm{cal} /(\mathrm{mol} \mathrm{K}))$, respectively. It is noteworthy that the enolization process in DMSO showed a remarkable decrease in the system entropy and enthalpy. It may be considered that this feature is attributed to the hydrogen bonding of PPA to the solvent DMSO molecule because the degree of the flexibility of both PPA and DMSO molecules are reduced or restricted due to strong intermolecular hydrogen bonding. It is also well known that the formation, such as a hydrogen bonding and van der Waals interaction, is an exothermic reaction and $\Delta H^{0}$ is negative. The results obtained in this study are qualitatively consistent with those of an ab initio calculation study previously reported; $\Delta H^{0}=-6.1$ $\mathrm{kcal} / \mathrm{mol}$ and $\Delta S^{0}=-7.7 \mathrm{cal} /(\mathrm{mol} \mathrm{K}){ }^{11}$

\section{Conclusions}

It was revealed by ${ }^{1} \mathrm{H}$ NMR measurement that PPA predominantly exist as the enol form in DMSO at room temperature. Increasing the temperature of this mixture increased the percentage of the keto form. The thermodynamic parameters for the tautomerism of PPA were experimentally obtained by the temperature-variable NMR measurements of this study. It is well known that a molar volume of a keto form is smaller than that of an enol form. It can thus be expected that $K_{\mathrm{t}}$ decreases as the pressure increases. It is worth carrying out a pressure-variable NMR measurement of PPA for a more detailed thermodynamic characterization of PPA in the future. Furthermore, the state-of-the-art calculation studies as directly taking account of a solute-solvent interaction for a solvent effect, for instance, by a QM/MM method would be also expected.

\section{References}

1. A. Meister, "Biochemistry of the Amino Acids", 1965, Vol. II, Academic Press, New York.

2. S. Sakurai, J. Biochem., 1956, 43, 851.

3. P. O. Larsen and E. Wieczorkowska, Acta Chem. Scand, Sect. B, 1974, 28, 92.

4. O. Sciacovelli, A. Dell'Atti, A. De Giglio, and L. Cassidei, Z. Naturforsch., 1976, 31c, 5.

5. L. Cassidei, A. Dell'Atti, and O. Sciacovelli, Z. Naturforsch., 1976, 31c, 641 .

6. L. Cassidei, A. Dell' Atti, and O. Sciacovelli, Z. Naturforsch., 1980, $35 c, 1$.

7. W. J. Ray, J. E. Katon, and D. B. Phillips, J. Mol. Struct., 1981, 74, 75.

8. K. Hanai, A. Kuwae, S. Kawai, and Y. Ono, J. Phys. Chem., 1989, 93, 6013.

9. K. Hanai, S. Kawai, and A. Kuwae, J. Mol. Struct., 1991, 245,21

10. T. Takai, H. Senda, H. H. Lee, A. Kuwae, and K. Hanai, Spectrosc. Lett., 1998, 31, 379.

11. A. J. M. Carpy, P. P. Haasbroek, J. Ouhabi, and D. W. Oliver, J. Mol. Struct., 2000, 520, 191.

12. D. W. Oliver, P. P. Haasbroek, J. M. Léger, and A. J. M. Carpy, J. Chem. Crystallogr., 1994, 24, 665.

13. H. H. Lee, K. Kimura, T. Takai, H. Senda, A. Kuwae, and K. Hanai, Spectrochim. Acta Mol. Biomol. Spectrosc., 1999, $55,2877$.

14. L. Onsager, J. Am. Chem. Soc., 1936, 58, 1486.

15. W. B. Bonnor, Trans. Faraday Soc., 1951, 47, 1143.

16. L. W. Reeves, Can. J. Chem., 1957, 35, 1351. 